\title{
Clinical significance of inferior ST elevation during acute anterior myocardial infarction
}

\author{
Akira Tamura, Hajime Kataoka, Kimiaki Nagase, Yoshiaki Mikuriya, Masaru Nasu
}

\begin{abstract}
Objectives-To clarify the genesis and clinical significance of inferior ST elevation during acute anterior myocardial infarction.
\end{abstract}

Patients and design-A total of 106 patients with first acute anterior myocardial infarction $(\leqslant 6 \mathrm{~h})$ were divided into two groups according to the presence (group $A, n=12$ ) or absence (group B, $\mathbf{n}=94$ ) of ST elevation of $\geqslant 1 \mathrm{~mm}$ in at least two of the inferior leads on the admission electrocardiogram.

Results-On admission electrocardiograms, group A had a smaller summed ST deviation in the lateral limb leads than group B. On emergency coronary arteriograms, the incidence of a wrapped left anterior descending artery was higher in group $A$ than in group B $(100 \% v 27 \%$, $P<0.01)$. The incidence of occlusion of a left anterior descending artery distal to its first diagonal branch was higher in group $A$ than in group $B(100 \% v 46 \%, P<0.01)$. Peak serum creatine kinase activity and in-hospital mortality tended to be lower in group $A$ than in group B. Group A had better left ventricular ejection fraction and regional wall motion in the anterobasal and anterolateral regions in the chronic phase than group B. In contrast, regional wall motion in the diaphragmatic region was reduced to a greater extent in group A than in group B.

Conclusions-Inferior ST elevation during acute anterior myocardial infarction appears only in the presence of a combination of a lesser degree of transmural ischaemic myocardium in the anterobasal and anterolateral wall together with transmural ischaemic myocardium in the inferior wall; in all cases there was occlusion of a wrapped left anterior descending artery distal to its first diagonal branch. Patients with such an ST elevation appear to have a better in-hospital prognosis than those without it.

(Br Heart f 1995;74:611-614)

Keywords: acute myocardial infarction; inferior ST elevation; prognosis after AMI

While the aetiology and clinical significance of inferior ST depression during acute anterior myocardial infarction have been investigated extensively, ${ }^{1-4}$ there are few reports ${ }^{56}$ concern- ing inferior ST elevation in such an infarction. Sapin $e t a l^{6}$ recently reported that inferior ST elevation during acute anterior myocardial infarction is commonly seen in patients with a distal occlusion of a left anterior descending coronary artery that extensively supplies the left ventricular inferior wall, the so-called wrapped left anterior descending artery. However, the clinical features and in-hospital prognosis of patients with inferior ST elevation during acute anterior myocardial infarction have not been fully studied. Our objective was to re-evaluate the aetiology of inferior ST elevation during acute anterior myocardial infarction and to clarify the clinical features and in-hospital prognosis of such patients.

\section{Methods}

PATIENTS

Between January 1989 and December 1992, 121 patients with acute anterior myocardial infarction were admitted to our hospital within $6 \mathrm{~h}$ of the onset of chest pain. Patients who met the following criteria were selected for this study: (1) typical chest pain lasting for at least $30 \mathrm{~min}$; (2) ST elevation of $\geqslant 2 \mathrm{~mm}$ in at least two adjacent precordial leads; (3) abnormal elevations of serum creatine kinase and of $M B$ creatine kinase activity; (4) no history or electrocardiographic evidence of previous myocardial infarction; (5) no electrocardiographic evidence of left ventricular hypertrophy, bundle branch block, or interventricular conduction disturbance (QRS $\geqslant 0.12 \mathrm{~s}$ ); (6) no primary valvar disease or myocardial disease; (7) no reperfusion treatment before emergency coronary angiography; (8) identification of the infarct related lesion by emergency coronary arteriography; and (9) angiographic visualisation of the entire distribution of the left anterior descending coronary artery following reperfusion treatment. There were 106 patients ( 80 men and 26 women, aged 36 to 81 years, mean 62 years) who met the inclusion criteria.

STANDARD 12-LEAD ELECTROCARDIOGRAMS Standard 12-lead electrocardiograms were obtained, being recorded at a paper speed of $25 \mathrm{~mm} / \mathrm{s}$ and a standardisation of $10 \mathrm{~mm}=1$ $\mathrm{mV}$. The magnitude of ST elevation or depression relative to the TP segment was measured to the nearest $0.5 \mathrm{~mm}$ at $80 \mathrm{~ms}$ after the J point. Measurements were obtained by the consensus of two observers who were blinded to all clinical and angiographic information. Inferior ST elevation was defined as 
Table 1 Clinical characteristics

\begin{tabular}{lccc}
\hline & Group $A$ & Group B & P value \\
\hline Age, years (SD) & $64(14)$ & $61(10)$ & NS \\
Men & $7(58 \%)$ & $73(78 \%)$ & NS \\
Time to ECG recordings, min (SD) & $189(75)$ & $154(76)$ & NS \\
Spontaneous recanalisation & $2(17 \%)$ & $12(13 \%)$ & NS \\
Good collaterals & $3(25 \%)$ & $16(17 \%)$ & NS \\
Multivessel disease & $5(42 \%)$ & $23(24 \%)$ & NS \\
Proximal LAD occlusion & $0(0 \%)$ & $51(54 \%)$ & $<0.01$ \\
Wrapped LAD & $12(100 \%)$ & $25(27 \%)$ & $<0.01$ \\
Methods of reperfusion & $10(83 \%)$ & $85(90 \%)$ & NS \\
$\quad$ PTCR & $6(50 \%)$ & $50(53 \%)$ & NS \\
PTCA & $1(17 \%)$ & $17(18 \%)$ & NS \\
Unsuccessful reperfusion & $1789(1339)$ & $2548(1844)$ & NS \\
Peak CPK, IU/l (SD) & $2(17 \%)$ & $11(12 \%)$ & NS \\
Reocclusion rate & $0(0 \%)$ & $6(6 \%)$ & NS \\
In-hospital death &
\end{tabular}

ECG, electrocardiogram; LAD, left anterior descending coronary artery; PTCR, percutaneous transluminal coronary revascularisation; PTCA, percutaneous transluminal coronary angioplasty; CPK, creatine kinase.
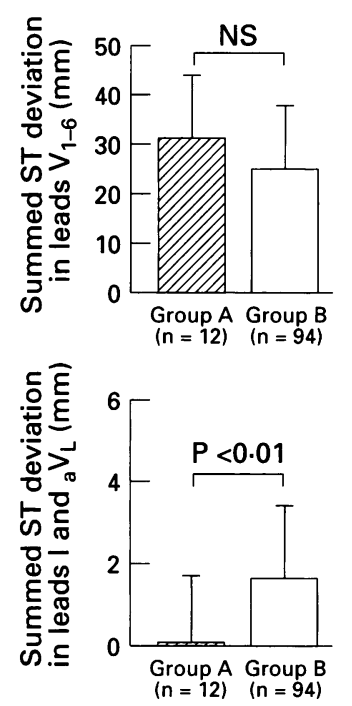

Sum of ST elevation or depression in leads other than aVR on admission electrocardiograms between the two groups. Group A: presence of ST elevation in inferior leads; group B: absence of ST elevation in inferior leads
ST elevation $\geqslant 1 \mathrm{~mm}$ in at least two of the inferior leads.

\section{CORONARY ANGIOGRAPHY AND REPERFUSION THERAPY}

Emergency coronary arteriography was' conducted using the Judkins or Amplatz technique. Multiple projections were recorded to ensure optimal visualisation of the coronary vessels. When the infarct related lesion was totally obstructed, we gave an intracoronary injection of isosorbide dinitrate followed by an intracoronary injection of urokinase or tissue plasminogen activator. If reperfusion could not be obtained or if there was severe residual stenosis, we performed coronary angioplasty. The grade of collateral filling in the left anterior descending artery was evaluated according to the criteria of Rentrop et al. ${ }^{7}$ A good collateral was defined as grade 2 or 3. A wrapped left anterior descending artery was defined as a left anterior descending artery that perfused at least one fourth of the inferior wall of the left ventricle. ${ }^{3}$ The establishment of reperfusion dial infarction trial, as grade 2 or 3 flow in the infarct related artery. ${ }^{8}$

\section{LEFT VENTRICULOGRAMS}

One month after the onset of acute myocardial infarction, the surviving patients underwent coronary arteriography and left ventriculography. Left ventriculograms were recorded in the 30 degree right anterior oblique and the 60 degree left anterior oblique view. End diastolic and end systolic endocardial contours were carefully traced by an experienced cardiologist unaware of the patient's clinical data. The global ejection fraction was calculated by the centreline method, and regional wall motion was expressed as standard deviation per was defined as in the thrombolysis in myocar-
Table 2 Left ventricular function in the chronic phase. Values are means (SD)

\begin{tabular}{lccc}
\hline & Group $A$ & Group B & Pvalue \\
\hline $\begin{array}{l}\text { Number of patients } \\
\text { Global LVEF (\%) }\end{array}$ & 12 & 88 & \\
RWM (SD/chord) & $59(11)$ & $51(12)$ & $<0.05$ \\
$\quad$ & $0.2(1.0)$ & $-1.3(0.9)$ & $<0.01$ \\
anterobasal & $-1.0(1.9)$ & $-2.3(1.5)$ & $<0.05$ \\
anterolateral & $-1.8(1.2)$ & $-1.6(1.5)$ & NS \\
apical & $-0.9(1.1)$ & $0.2(1.3)$ & $<0.05$ \\
\hline
\end{tabular}

LVEF, left ventricular ejection fraction; SD, standard deviation; RWM, regional wall motion. chord. ${ }^{9}$ The anterobasal, anterolateral, apical, or diaphragmatic area was defined as chord number 1 to 20,21 to 40,41 to 60 , or 61 to 80 , respectively.

\section{STATISTICS}

Data are expressed as mean (SD). Categorical data were analysed by Fisher's exact test or the $\chi^{2}$ test with Yates' correction. Continuous variables were analysed by the unpaired $t$ test. A $P$ value $<0.05$ was considered statistically significant.

\section{Results}

Admission electrocardiograms showed that 12 patients had inferior ST elevation (group A), while 94 patients did not (group B). The two groups did not differ as to age, gender, time elapsed from the onset of infarction to the electrocardiographic recording, the success rate of reperfusion therapy, or the rate of reocclusion (table 1).

\section{ST ELEVATION IN THE PRECORDIAL AND LATERAL LIMB LEADS ON ADMISSION ELECTROCARDIOGRAMS}

While the sum of ST deviation in the precordial leads on the admission electrocardiograms did not differ significantly between the two groups, at 31 (SD 13) $\mathrm{mm}$ versus 25 (12) $\mathrm{mm}$, the sum of ST deviation in the lateral limb leads was smaller in group $A$ than in group B: $0(1.7) \mathrm{mm} v 1.7(1.8) \mathrm{mm}, \mathrm{P}<$ 0.01 (figure).

EMERGENCY CORONARY ANGIOGRAMS (table 1) The incidence of a wrapped left anterior descending artery was higher in group $\mathrm{A}$ than in group $\mathrm{B}(100 \% v 27 \%, \mathrm{P}<0.01)$. When we investigated the site of the occlusion of the left anterior descending artery, group A showed a higher incidence of occlusion of the left anterior descending artery distal to its first diagonal branch than group B $(100 \% v 46 \%$, $\mathrm{P}<0.01$ ).

\section{CLINICAL VARIABLES (table 1,2 )}

Peak serum creatine kinase values tended to be lower in group A than in group B, at 1789 (1339) v 2548 (1844) IU/l, NS. In-hospital mortality tended to be lower in group $A$ than in group B $(0 \% v 6 \%, \mathrm{NS})$. Global left ventricular ejection fraction in the chronic phase was significantly greater in group $A$ than in group $\mathrm{B}$, at $59(11) \% v 51(12) \%, \mathrm{P}<0.05$. Regional left ventricular wall motion in the anterobasal and anterolateral regions was less reduced in group A than in group B: $0 \cdot 2(1 \cdot 0)$ $v-1.3(0.9), \quad \mathrm{P}<0.01 ; \quad$ and $-1.0(1.9) \quad v$ $-2 \cdot 3(1 \cdot 5), P<0.05$, respectively. In contrast, regional wall motion in the diaphragmatic region was more reduced in group $A$ than in group $\mathrm{B}:-0.9(1.1) v 0.2(1.3), \mathrm{P}<0.05$.

\section{Discussion}

We investigated the incidence of a wrapped left anterior descending coronary artery in patients with acute anterior myocardial infarc- 
tion and found that it was present in all 12 patients with inferior ST elevation. This indicates that the transmural ischaemia in the inferior wall caused by the occlusion of a wrapped left anterior descending artery is a prerequisite for inferior ST elevation during acute anterior myocardial infarction. However, 25 of the 37 patients with a wrapped left anterior descending artery (68\%) did not show inferior ST elevation. Thus occlusion of a wrapped left anterior descending artery is not the only cause of inferior ST elevation. We noted that patients with inferior ST elevation had smaller ST elevation in the lateral limb leads than those without it, and hypothesised that the absence of ischaemic involvement of the first diagonal branch may be related to inferior ST elevation during acute anterior myocardial infarction. Transmural ischaemia in the region perfused by the first diagonal branch would produce ST elevation in the lateral limb leads, ${ }^{10}$ resulting in simultaneous reciprocal ST depression in the inferior leads. Therefore, even though transmural ischaemia occurs in both the anterior and inferior walls due to the occlusion of a wrapped left anterior descending artery, if the force of the injury current produced by transmural ischaemia in the lateral wall exceeds that produced by transmural ischaemia in the inferior wall, inferior ST elevation would be absent. Indeed, all patients with inferior ST elevation in this study had an occlusion of the left anterior descending artery distal to its first diagonal branch. Thus the absence of ischaemic involvement of the first diagonal branch is a requisite for the inferior ST elevation during acute anterior myocardial infarction.

In our study, nine of the 21 patients with occlusion of a wrapped left anterior descending artery distal to its first diagonal branch (43\%) had no inferior ST elevation. All those patients had an occlusion of the left anterior descending artery between its first and second diagonal branches and showed ST elevation of $\geqslant 1 \mathrm{~mm}$ in leads I and aVL. In these patients, the reciprocal force of the lateral ST elevation produced by transmural ischaemia in the area perfused by the second diagonal branch would have overcome the inferior ST elevation produced by transmural ischaemia in the inferior wall.

Another angiographic feature can cause inferior ST elevation during acute anterior myocardial infarction. Occlusion of a left anterior descending artery supplying the left ventricular inferior wall through collateral channels to an occluded right or dominant circumflex coronary artery can produce anterior wall ischaemia combined with inferior wall ischaemia, resulting in inferior ST elevation accompanied by precordial ST elevation. However, this situation was not found in any patient in our study.

There are no previous reports on the inhospital outcome of patients with inferior ST elevation during acute anterior myocardial infarction. In our study, the peak serum creatine kinase values and in-hospital mortality rates tended to be lower in the patients with than in those without inferior ST elevation. Left ventriculograms obtained in the chronic phase showed that patients with inferior ST elevation had significantly better left ventricular ejection fraction and regional wall motion in the anterobasal and anterolateral regions than those without it, although regional wall motion in the diaphragmatic region was more reduced in the patients with inferior ST elevation. These findings indicate that patients with acute anterior myocardial infarction and inferior ST elevation appear to have a relatively good in-hospital prognosis. These results appear to be reasonable, considering the angiographic morphology that can lead to inferior ST elevation.

In acute anterior myocardial infarction, patients with inferior ST depression have a worse prognosis than those without it. ${ }^{1}$ In contrast the present study indicates that the patients with inferior ST elevation appear to have a relatively better in-hospital prognosis. Thus analysis of inferior ST deviation on admission electrocardiograms may be useful in evaluating the severity and prognosis of acute anterior myocardial infarction.

Our study has some limitations. First, most of our patients received reperfusion treatment. It has been shown that early reperfusion treatment in acute myocardial infarction can limit the size of the infarct, preserve left ventricular function, and improve survival. ${ }^{11}$ Therefore our data may not reflect the natural history of patients with acute anterior myocardial infarction showing inferior ST elevation with respect to infarct size or prognosis. Second, relatively few of our patients had inferior ST elevation, and the phenomenon appears to be relatively rare. Further studies in a larger population are desirable to confirm the present results.

In conclusion, this study shows that inferior ST elevation during acute anterior myocardial infarction appears only in the setting of a combination of a lesser degree of transmural ischaemic myocardium in the anterobasal and anterolateral wall and the simultaneous presence of transmural ischaemic myocardium in the inferior wall; in all cases there was occlusion of a wrapped left anterior descending artery distal to its first diagonal branch. Patients with such an ST elevation appear to have a relatively better in-hospital prognosis.

1 Haraphongse $M$, Tanomsup S, Jugdutt BI. Inferior ST segment depression during acute anterior myocardial infarction: clinical and angiographic correlations. $\mathcal{f} \mathrm{Am}$ Coll Cardiol 1984;4:467-76.

2 Quyyumi AA, Rubens MB, Rickards AF, Crake T, Levy $\mathrm{RD}$, Fox KM. Importance of reciprocal electrocardiographic changes during occlusion of left anterior descending coronary artery. Lancet 1986;i:347-50.

3 Lew AS, Hod H, Cercek B, Shah PK, Ganz W. Inferior ST segment changes during acute anterior myocardial infarction: a marker of the presence or absence of concomitant inferior wall ischemia. $₹ \mathrm{Am}$ Coll Cardiol 1987;10:519-26.

4 Norell MS, Lyons JP, Gardener JE, Layton CA, Balcon R. Significance of "reciprocal" ST segment depression: left ventriculographic observations during left anterior descending 1989;13:1270-4.

5 Ilia R, Goldfarb B, Ilia A, Ovsyshcher IA. Concomitant ST elevation in inferior and anterior leads in acute myocardial infarction. $\mathcal{F}$ Electrocardiol 1990;23:199-205.

6 Sapin PM, Musselman DR Depmer G Im PM, Musselman DR, Dehmer GJ, Cascio WE. nying anterior wall acute myocardial infarction for the 
angiographic morphology of the left anterior descending coronary artery morphology and site of occlusion. $\mathrm{Am} \mathrm{F}$ Cardiol 1992;69:860-5.

7 Rentrop KP, Cohen M, Blanke H, Phillips RA. Change in collateral channel filling immediately after controlled coronary artery occlusion by an angioplasty balloon in
human subjects. $₹ \mathrm{Am}$ Coll Cardiol 1985;5:587-92.

8 The TIMI study group. The Thrombolysis in Myocardial Infarction (TIMI) trial: phase I findings. $N$ Engl $f$ Med 1985;312:932-6.

9 Sheehan FH, Bolson EL, Dodge HT, Marthey DG,
Schofer J, Woo HW. Advantages and applications of the centerline method for characterizing regional ventricular function. Circulation 1986;74:293-305.

10 Iwasaki K, Kusachi S, Kita T, Taniguchi G. Prediction of isolated first diagonal branch occlusion by 12 -lead electrocardiography: ST segment shift in leads I and aVL. $\mathcal{F}$ trocardiography: ST segment shift in leads $I$ and aVL. $\mathcal{F}$

11 Simoons ML, Serruys PW, Brand M, Res J, Verheug FWA, Krauss XH, et al. Early thrombolysis in acute myocardial infarction: limitation of infarct size and improved survival. $\Im$ Am Coll Cardiol 1986;7:717-28. 